\title{
Investigating the components of signals produced by the Pockels CCD imaging technique in CdZnTe
}

\author{
S. Parsons, ${ }^{1,2}$ A. Langley, ${ }^{2}$ C. Shenton-Taylor, ${ }^{2}$ A. Lohstroh ${ }^{1}$ \\ ${ }^{1}$ Department of Physics, University of Surrey, Guildford, GU2 7XH, United Kingdom \\ ${ }^{2} A W E$ Aldermaston, Reading, RG7 4PR, United Kingdom
}

\section{ABSTRACT}

Several groups have demonstrated the potential of the Pockels effect in Cadmium Zinc Telluride (CZT) as a means to detect ionizing radiation. Migrating charge carriers are believed to generate the signal detected via the Pockels effect due to the distortions they create within the electric field, however trapped space charge beneath the cathode has been regularly observed which suggests field changes away from the charge generation area. Consequently the Pockels signal amplitude contains a charge integrated, i.e. dose element. In this work, the effects of electric field collapse at the location of charge carrier generation are demonstrated. This opens the potential to apply the technique for imaging dose rate distributions. Charged coupled device (CCD) images representing the changes in electric field within the crystal were taken and the response to illumination from a collimated $1550 \mathrm{~nm} 4.5 \mathrm{~mW}$ IR laser and irradiation from $150 \mathrm{kVp}$ X-rays measured. The data demonstrates that the signal acquired is a combination of both the local change in the electric field at the location where the carriers are being released/generated and an element caused by carriers becoming trapped, leading to electric field enhancement due to space charge near the cathode, and field reduction towards the anode.

\section{INTRODUCTION}

A typical semiconductor detector for ionizing radiation converts the energy deposited by incident radiation into free charge carriers, which can then be collected to give information on the characteristics of the ionization event. In high flux environments, electronic components can become damaged and signals induced in connecting cables due to the proximity of the radiation field. The amplification stages are typically positioned close to the detectors photosensitive region to minimize line losses and input capacitance from the connecting wires.

This paper discusses work on the Pockels detection technique which observes how the charges created in an ionization event affect the electric field within Cadmium based compounds. Experiments by Nelson et al. demonstrated that information on a high intensity neutron/gamma flux from a reactor pulse irradiating a Cadmium Zinc Telluride (CZT) crystal can be gathered by measuring the changes in the electric field [1]. Subsequent experiments by Prekas et al. showed a similar capability with X-ray radiation [2]. The main advantage of this approach is that the radiation sensitive electronics can be positioned a long distance from the radiation field as collected charge is not being processed; this potentially means a system built using this technology could be left in-situ within a high flux environment as minimum maintenance would be required. In a similar way to detectors running in continuous current mode, the system should not suffer from dead time as there is no photon counting, thereby removing the associated signal processing overheads and making it possible to operate in higher flux intensities. However, it is likely that detectors in pulse mode are more sensitive due to a lower detection limit $[1,3]$.

The technique utilizes the electro-optical property known as Pockels effect, where the refractive index of a material varies with the strength of the electric field it experiences. The presence of charges within the medium can distort the electric field, creating variation in the refractive index which is observed by recording how the polarization direction of transmitted light changes.

In isotropic binary and tertiary crystals such as Cadmium Telluride (CdTe), CZT, or Cadmium Manganese Telluride (CMT), application of an electric field causes the material to become uniaxial whereby it has two distinct refractive indexes with one perpendicular to the field lines and the other parallel. The index on the latter (known as the optic axis) is proportional to the field strength as shown in (1) where $E$ is the electric field strength, $n(E)$ is the modified refractive index, $n_{0}$ is the field free refractive index at wavelength $\lambda$ and $r_{41}$ is the Pockels coefficient.

$$
n(E)=n_{0}+\left(\frac{1}{2}\right) n_{0}^{3} r_{41} E
$$

The majority of work within this area has examined CZT crystals which, due to their inherent properties, have low hole mobility. In addition, the material typically contains a sufficiently high concentration of electrically active defects to allow the crystal to build up space charge via the trapping of free charge carriers. This space charge, changes the local electric field and hence the refractive index. As such it is possible that the majority of the detected Pockels signal is formed this way, especially if measured close to the cathode where the electric field strength is enhanced $[2,4,5]$. If this is the case then the change in refractive index will have a potentially large time integrated dose element; with a time scale determined by the re-emission of trapped charges from the defect states. However, the local photogeneration of free charge carriers is also expected to affect the electric field

Page 1 of 8 
distribution but in a less accumulative manner. This photogeneration introduces a variation in the free charge carrier density throughout the crystal that is likely to accurately follow the dose rate distribution incident on the material. The potential presence of both of these effects has been acknowledged in a previous study [6] however they have not been explicitly identified independently. The study discussed within [6] used a $1550 \mathrm{~nm}$ laser to illuminate the entire CZT crystal and then imaged the electric field via a CCD camera combined with a pair of crossed polarizers. Measurements were captured with the cathode at both the top and bottom of the crystal for both bias polarities, with the results showing a build-up of space charge under the cathode in either bias direction, even when the sample was only irradiated near the anode side. This indicated that polarization is contributing to the detected signal. Our current work has focused entirely on investigating the detected Pockels signal, separating the space charge aspects across the whole sample volume from the signal component created at the charge carrier generation location. Being able to understand the relationship between the two elements could lead to taking real-time measurements with this technique which would provide the potential to image radiation fields within the crystal.

\section{EXPERIMENTAL METHOD}

In order to assess whether the signal contains a local element we used the same setup as in [6], see Figure 1, with the exception of a $1 \mathrm{~cm}^{3}$ Redlen CZT sample as a detection medium which has one gold planar electrode and a co-planar grid wired together to act as a planar electrode to create a uniform electric field within the crystal. The imaging system works by illuminating the crystal with linearly polarised light which has been set to $-45^{\circ}$ with respect to the electric field applied to the CZT by a linear polariser and then via a $2^{\text {nd }}$ linear polariser set at $+45^{\circ}$ extracting the components of the beam which have had their polarities changed whilst passing through the birefringent medium. The large crystal enabled us to illuminate / irradiate the side with a collimated laser or an $\mathrm{X}$-ray beam to observe any changes in the electric field that it caused. A $4.5 \mathrm{~mW}, 1550 \mathrm{~nm}$ laser from Thorlabs (LDM1550) was collimated into a $1 \times 10 \mathrm{~mm}^{2}$ beam and a $3 \mathrm{~kW}$ Comet Xray tube with a tungsten target (MXR-225/22), this was collimated via lead bricks into a $1 \times 20 \mathrm{~mm}^{2}$ beam. Although NIR light can only photogenerate charge carriers from energy levels within the bandgap resulting in free charge carriers [7], it is expected that the effect of photogeneration of charge carriers caught in mid-bandgap traps on the electric field using a NIR laser beam is similar to that caused by $\mathrm{e}-\mathrm{h}$ pair generation from X-rays [8]. The main differences are the lower energy and mono-energetic nature of the photons which will cause the beam to penetrate further into the crystal as it is sub-band gap. The X-ray beam has not been characterised explicitly for this work however it is expected that the spatial intensity distribution will be approximately Gaussian.

The light intensity transmitted through the second polarizer $(I)$ is related to the electric field strength $(E)$ by (2) where $\lambda$ is the wavelength of the light, $I 0$ is the intensity from the light source and $d$ is the length of the light path through the crystal [2].

$$
I=I_{0} \sin ^{2}\left(\frac{\sqrt{3} \pi n_{0}^{3} r_{41} d}{2 \lambda} E\right)
$$

For both the X-ray and laser sources, the distance between the collimator and crystal was $\sim 5 \mathrm{~cm}$ and from the source to collimator $\sim 10 \mathrm{~cm}$, leading to a total of $\sim 15 \mathrm{~cm}$ from the crystal to the source in the laser setup and $\sim 30 \mathrm{~cm}$ in the X-ray setup due to the additional thickness of the collimator. In order to record the localized response to the photogeneration of charge carriers, the $1 \times 10 \mathrm{~mm}^{2}$ laser beam was scanned along the $\mathrm{x}$-axis of the crystal in $0.50+/-0.01 \mathrm{~mm}$ increments using a micrometer controlled translation stage. To investigate sensitivity to variation in intensity of the laser, the width and power output of the laser beam were varied and the FWHM/peak absolute magnitude of the dip recorded.

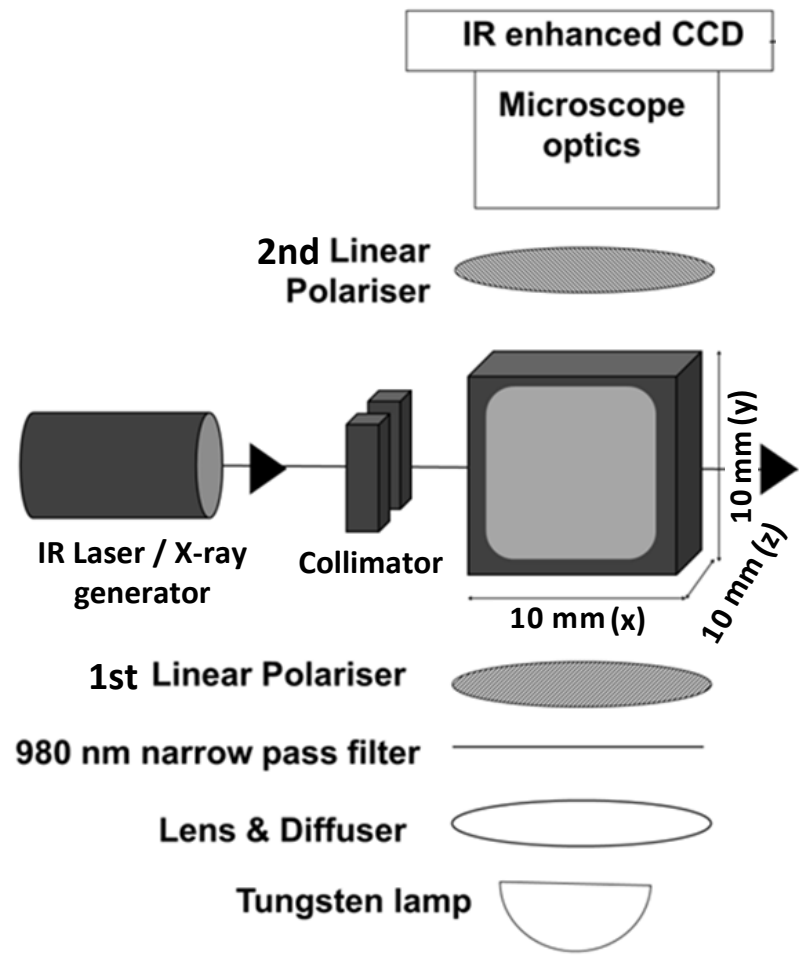

Figure 1: Schematic of the Pockels imaging setup used to acquire images under IR laser and X-ray illumination / irradiation, adapted from [6].

Whilst varying the width, the initial power output of the laser was decreased as the collimator was widened to maintain a constant level of overall optical power $(0.5 \mathrm{~mW})$ incident on the crystal. This was monitored via an Ophir 3A-P laser sensor connected to a Nova 2 energy meter. The power of the laser was varied using a set of neutral density filters. The bias voltage was set at $-2.00 \mathrm{kV}$ when the top contact was the cathode and $1.75 \mathrm{kV}$ when making it the anode. This corresponds with the crystal quarter wave point, illustrated in Figure 2, where the Pockels detection technique is most sensitive to changes in refractive index [1]. A $\sin ^{2}$ fit described 
the data well as expected from equation (2) and provided $r_{41}$ values of $6.62+/-0.03 \times 10^{-12} \mathrm{~m} / \mathrm{V}$ and $7.21+/-0.03 \times 10^{-12}$ $\mathrm{m} / \mathrm{V}$ for negative and positive bias respectively. This value is slightly higher than the literature values of $4.5-5.0 \times 10^{-12} \mathrm{~m} / \mathrm{V}$ but still of the same order [9]. Furthermore, results reported by Schwartz et al. showed that the coefficient may be strongly dopant dependent hence the difference could be caused by the presence of small levels of impurities in the crystal [10]. The current-voltage curve is asymmetric which hints that the sample does not behave exactly the same under both positive and negative bias. This potentially also begins to explain the difference in the positive and negative Pockels curves shown in Figure 2 which have been created by averaging along the $x$ and $\mathrm{z}$ directions of the crystal, where the slope gradients are almost the same, but the two curves are offset in the voltage position.

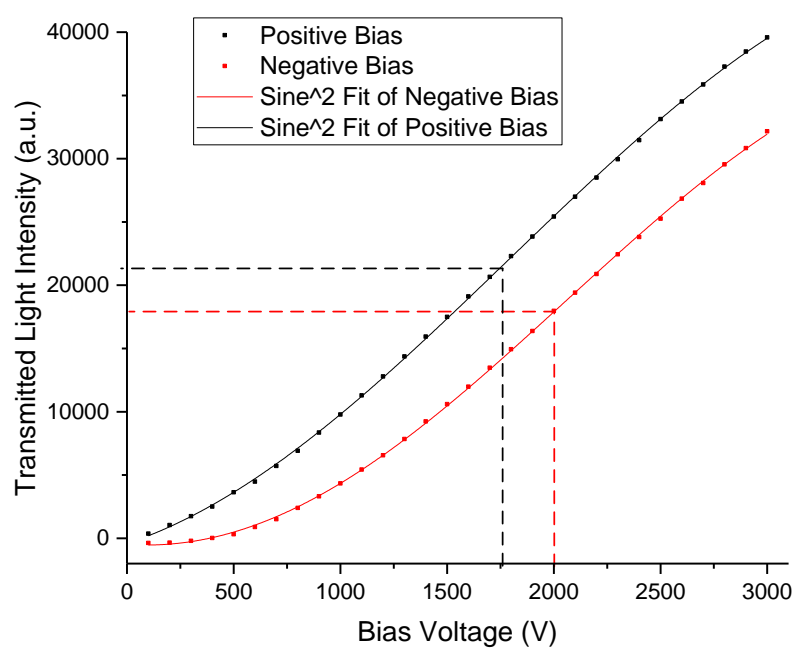

Figure 2: Shows the Pockels curve and the quarter wave point for the CZT crystal with a negative and positive bias applied to the top planar electrode.
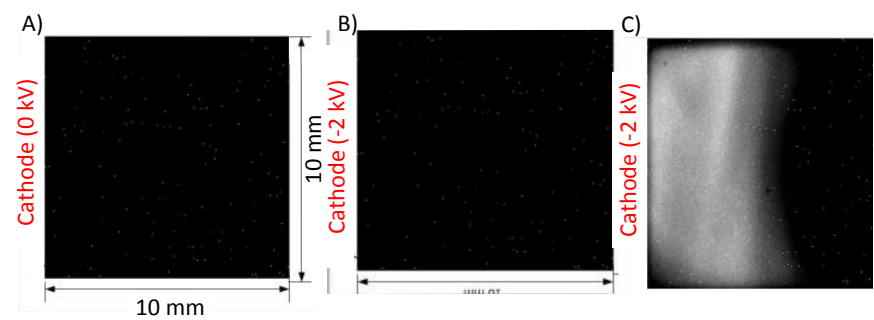

Figure 3: Raw Pockels images of $1 \mathrm{~cm}^{3}$ Redlen CZT crystal with A) $0.00 \mathrm{~V}$ bias applied to the top electrode (left side in figure), B) $-2.00 \mathrm{kV}$ bias and C) $2.00 \mathrm{kV}$ bias with entire crystal laser illumination.

The CCD light transmission images were captured via a GUPPY F-044B NIR CCD camera with a 4 second acquisition time. It can be seen in Figure $3 \mathrm{~A}$ that there is little light transmitted through the crystal at $0.00 \mathrm{~V}$ bias which indicates that the level of stress induced birefringence caused by any defects is minimal [11]. The system is designed to minimize light from the probing beam of the lamp producing charges in the crystal; a dark room is also used to reduce light from external sources. As the technique creates an image using a 4 second acquisition time, which has been selected due to the low intensity of the light being transmitted (higher intensity light from the lamp causes trapped space charge to be generated), and as the carrier lifetimes are significantly shorter ( 16-160 $\mu$ s for electrons and 3-30 ns for holes [4]), the measurement does not capture the fast component of the signal. This means that the data is not sensitive directly to the changes in electric field as the carriers drift to the electrodes but, instead, it observes a trend averaged over the acquisition time. 


\section{RESUlTS}

\section{A. Correlation of signal position and incident illumination position (IR)}

To test whether local changes in the electric field can be detected a collimated laser beam was used to illuminate the crystal and the resulting changes in light transmission recorded by the CCD and converted into $\mathrm{V} / \mathrm{cm}$ via (2). The profile plots seen in Figure 4 show how there is a detectable change in the field strength as the beam is scanned down the crystal. The plots were generated by subtracting one image with only the bias voltage applied (Figure 3B) from one with the bias and the laser/X-ray (Figure $3 \mathrm{C}$ ) activated. This was completed to counteract any non-uniformity present in the birefringence due to crystal geometry, defects etc. The images used for $I_{0}$ in all of the results were taken at a later date and whilst the same setup was used the alignments were not exactly the same which may have caused the values for electric field strengths in Figures 4, 6 and 10 to be smaller than expected for a $2 \mathrm{kV}$ bias. The results still show however that a localized birefringence effect is detectable. As the laser is scanned down the crystal there is a clear change in electric field strength where the laser beam photogenerates the carriers.

A)
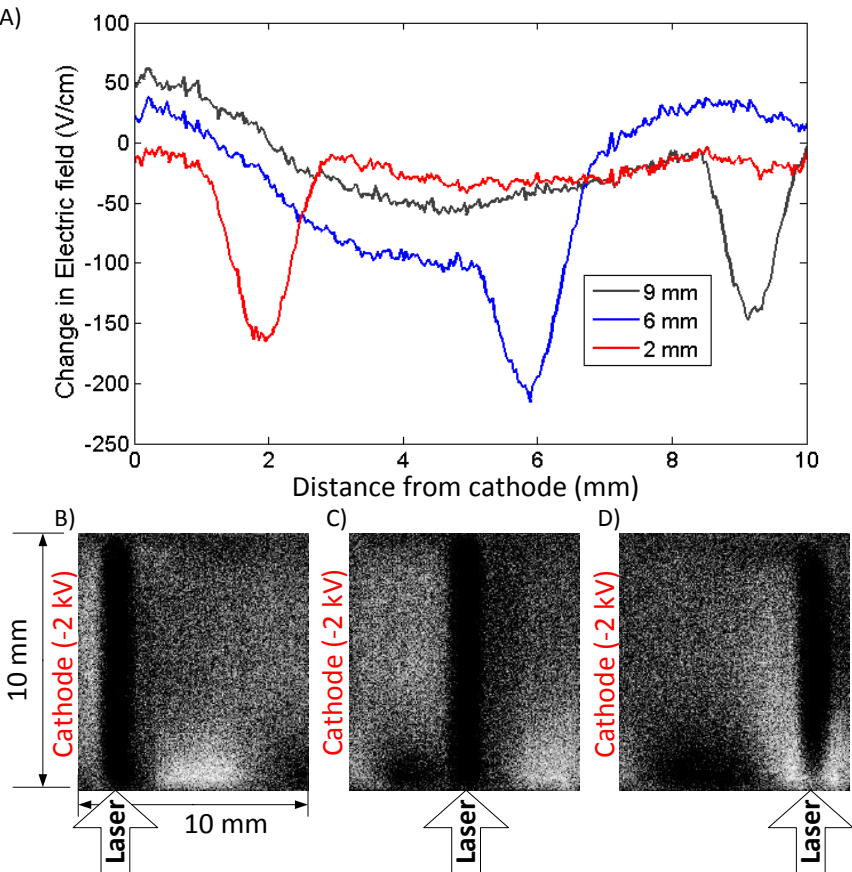

Figure 4: A) Displays the local changes in background corrected transmitted light intensity profiles within a CZT crystal under $-2.00 \mathrm{kV}$ bias caused by illuminating with a collimated $1550 \mathrm{~nm}$ laser beam at a variety of different beam positions in respect to the cathode (images are rotated by $90^{\circ}$ to provide clarity on laser position). B) CCD light transmission image with laser at 2.0 $\mathrm{mm}$. C) Laser at $6.0 \mathrm{~mm}$. D) Laser at $9.0 \mathrm{~mm}$ distance from the anode.

\section{B. Confirming that incident beam widths correlates to} signal width and signal intensity to illumination power

With the presence of local changes confirmed, the sensitivity to changes in the laser beams width and power were investigated to provide an indicator of whether this technique will be capable of detecting this type of variation.

The results (Figure 5) show that as the collimated beam widens the FWHM of the transmitted light distribution increases, excluding the data point at $0.5 \mathrm{~mm}$ which is caused by Fraunhofer diffraction through the collimator thereby giving an effective beam width of $\sim 1.5 \mathrm{~mm}$ incident on the crystal [12]. The same relationship is seen when measured at different points throughout the crystal. A linear fit with $95 \%$ confidence bounds is displayed in Figure 5, which encapsulates all the data points within a 1 standard deviation error.

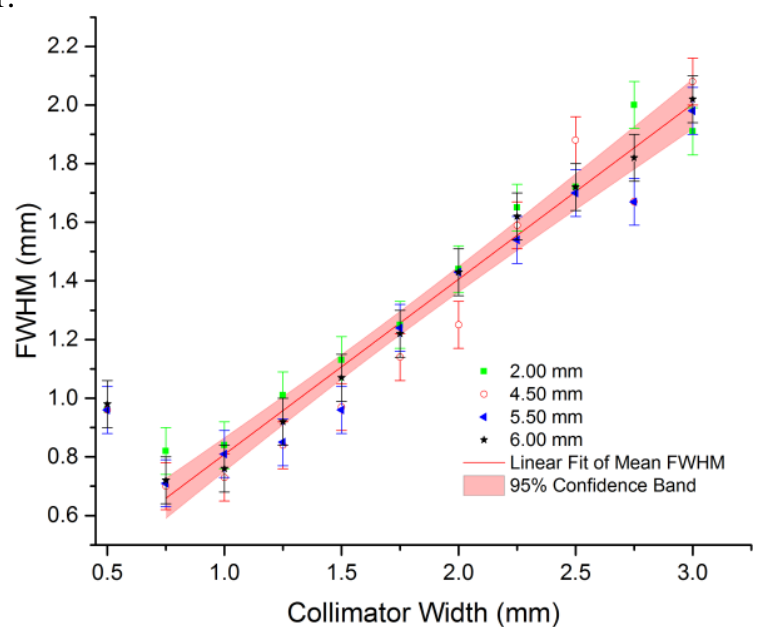

Figure 5: Variation in FWHM of intensity dip with increasing collimator width at different points in the crystal under $-2 \mathrm{kV}$ bias, error bars are calculated from the Gaussian fits of the peaks.

This combined with the results in Figure 6, where a linear relationship between the power of the laser and the peak light intensity was measured, means that not only will any detected signal from a laser beam contain a locally produced element it will also be sufficiently sensitive to it so that changes in the radiation/illumination beam intensity and position can be detected. The outlier highlighted in the FWHM plot of Figure 6 was attributed to the low power of the laser, causing the intensity dip to be very close to the noise floor. This meant that while an absolute intensity could be estimated, the peak was not symmetrical, so a Gaussian fit and hence the FWHM measurement was very sensitive to the choice of fit region causing the result to become distorted. It is expected that if the peak could have been resolved accurately, the FWHM value would have been similar to those at higher powers. In addition the error bars on that point are also too small as it is calculated via the Gaussian fit; uncertainties of approximately $+/-0.5$ $\mathrm{mm}$ are more realistic for this data point. 


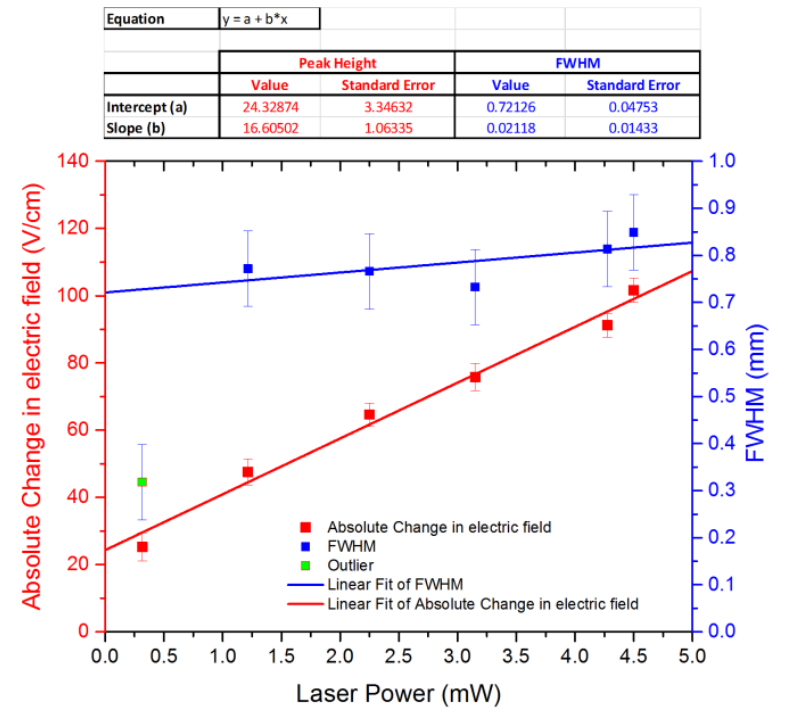

Figure 6: Variation in the depth of intensity dip (measured at $4.5 \mathrm{~mm}$ from cathode) with increasing laser power (red) under $-2.00 \mathrm{kV}$ bias, the FWHM remains constant (blue).

\section{Broad beam X-ray illumination}

This Pockels detection technique is intended to be used with ionizing radiation; therefore parts of the experiment were repeated using a collimated X-ray beam. As a first step the entire crystal was irradiated by $150 \mathrm{kVp}$ X-rays at $5 \mathrm{~mA}$ to investigate whether the electric fields response would be uniform.

The results in (Figure 7B and $\mathrm{C}$ ) show that fringes of high and low light transmission are created beneath the cathode, this is caused by a large electric field in the area and as seen in (2), the transmitted light has a sinusoidal relationship with the field strength which is likely what creates this; a similar effect is also reported by Cola [13]. These features are created by the accumulation of space charge, which we cannot see (clearly) in the laser data.

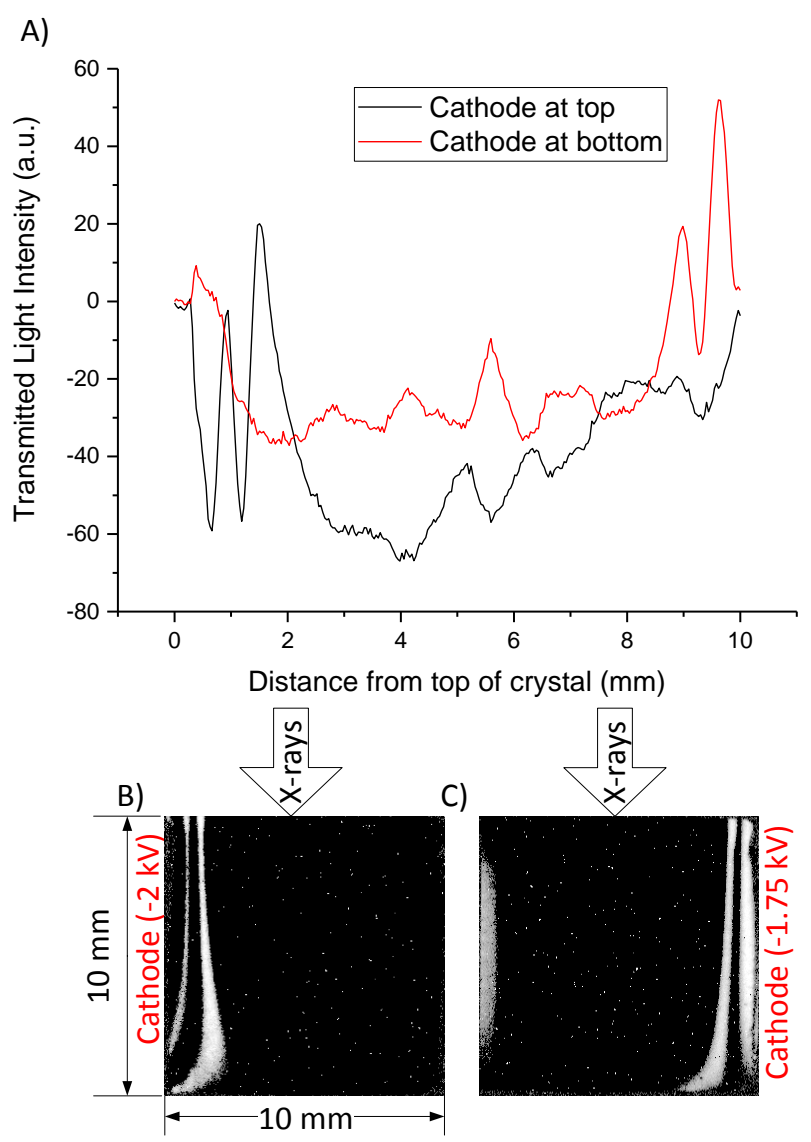

Figure 7: A) Transmitted light intensity profiles under entire crystal X-ray irradiation with cathode at either end of the crystal. B) CCD light transmission image with $-2.00 \mathrm{kV}$ applied to the top electrode. C) CCD light transmission image with $-1.75 \mathrm{kV}$ applied to the bottom electrode.

\section{Correlation of collimated $X$-ray position and signal position}

To confirm that the local effects from the charge carriers generated using the IR laser also occur with X-ray induced free charge carriers, a $1 \mathrm{~mm}$ collimated X-ray source was compared to the laser illumination of the crystal. The beam was positioned at the $5 \mathrm{~mm}$ point and varied over a range of $\mathrm{X}$-ray tube currents. Unlike when using the collimated laser, there is clear enhancement of the crystals electric field beneath the cathode and at locations away from where the collimated $\mathrm{X}$-ray beam is focused which creates a CCD image similar to that shown in Figure 8C where the crystal displays lower light transmission nearer the anode and higher area around the cathode due to the building up of space charge and the subsequent collapse of the electric field. This is likely due to a combination of there being significantly more free carriers generated with the X-rays than with IR. It is possible to analyse this type of image by removing a variable baseline generated by tracing the $0.1 \mathrm{~mA}$ profile along with the 2.00 $\mathrm{kV}$ background image to isolate the area around the incident $\mathrm{X}$-rays and to enable fitting a Gaussian distribution, as seen in Figure $8 \mathrm{~A}$ and $\mathrm{B}$. 

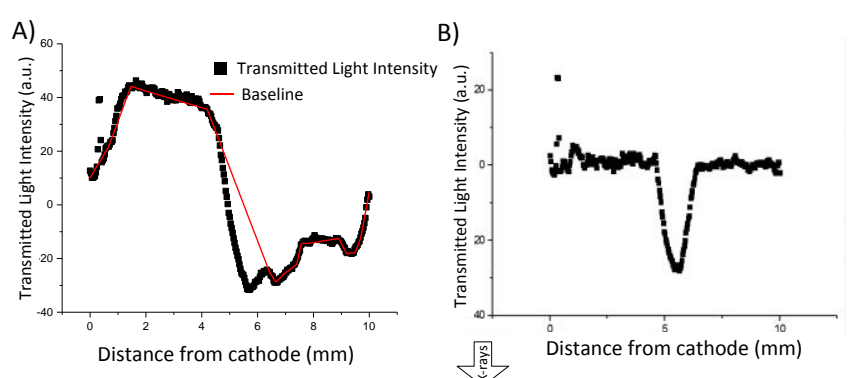

C)

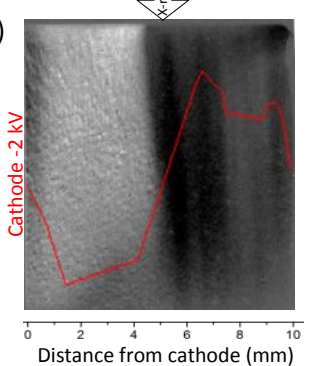

Figure 8: A) Light transmission profile under $0.4 \mathrm{~mA}, 150 \mathrm{kV}$ X-ray irradiation at $5 \mathrm{~mm}$ below cathode, an example of the type of baseline used is shown in red. B) Resulting Gaussian peak from baseline subtraction. C) CCD image of crystal under irradiation with baseline overlaid

However, clearer images are achieved if an image under bias and a low X-ray flux is subtracted (images with an X-ray tube current of $0.2 \mathrm{~mA}$ are used and should not be confused with the $0.1 \mathrm{~mA}$ images used for the baselining in Figure 8) as this reduces the scattering effects so that it cannot be as easily distinguished from noise anymore and enables the location of the collimated X-ray beam to be identified easily, an example of this is seen in Figure 9. A light transmission profile plot such as in Figure 9B can then be generated by averaging the images along $\sim 3.5 \mathrm{~mm}$ of the $\mathrm{Y}$-axis (corresponding to the 1/e intensity drop $=3.55 \mathrm{~mm}$ of the $150 \mathrm{kV}$ X-rays, based on a mass-attenuation coefficient $=4.852 \times 10^{-1} \mathrm{~cm}^{2} / \mathrm{g}$ and a CZT density $=5.8 \mathrm{~g} / \mathrm{cm}^{3}$ ); negative values on the Y-axis are areas of low light transmission (dark areas in images) [14]. The poly-energetic nature of the X-ray beam means that the majority of the photons will be below $150 \mathrm{keV}$ and as such the value used for the mass-attenuation coefficient is an over estimation. This means that most of the X-rays will not penetrate beyond the $1 / \mathrm{e}$ point, however the coefficient has been chosen as it corresponds to the maximum energy and hence penetration depths possible from this beam line and as seen in the figures the interactions take place approximately where predicted.
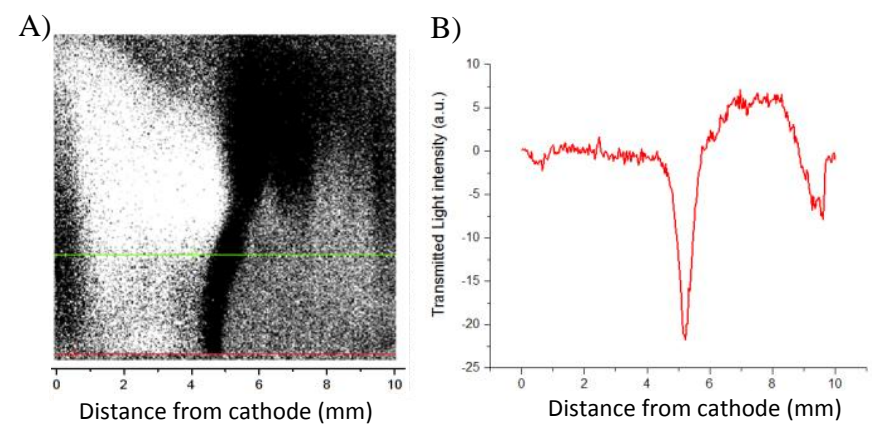

Figure 9: A) CCD image of crystal under irradiation at an X-ray tube current of $0.6 \mathrm{~mA}$ with $0.2 \mathrm{~mA}$ image subtracted (area between red and green markers is the section analysed) B) Resulting light transmission profile

\section{E. Correlation between $X$-ray irradiation intensity and signal intensity}

To confirm that the signal response to collimated X-rays is sensitive to changes in beam intensity (which would therefore mean that charge carrier trapping is not dominant on the length scales we are looking at) the X-ray tube current was varied between $0-10 \mathrm{~mA}$ and the change in amplitude of the signal response recorded. Between 0-1.0 mA a linear fit has been applied to the data in Figure 10 as this is the expected behavior as the absolute peak light intensity varies with the current. However, unlike the laser results, it does deviate from the linear fit and as can be seen in the inset the intensity starts to plateau beyond $1 \mathrm{~mA}$.

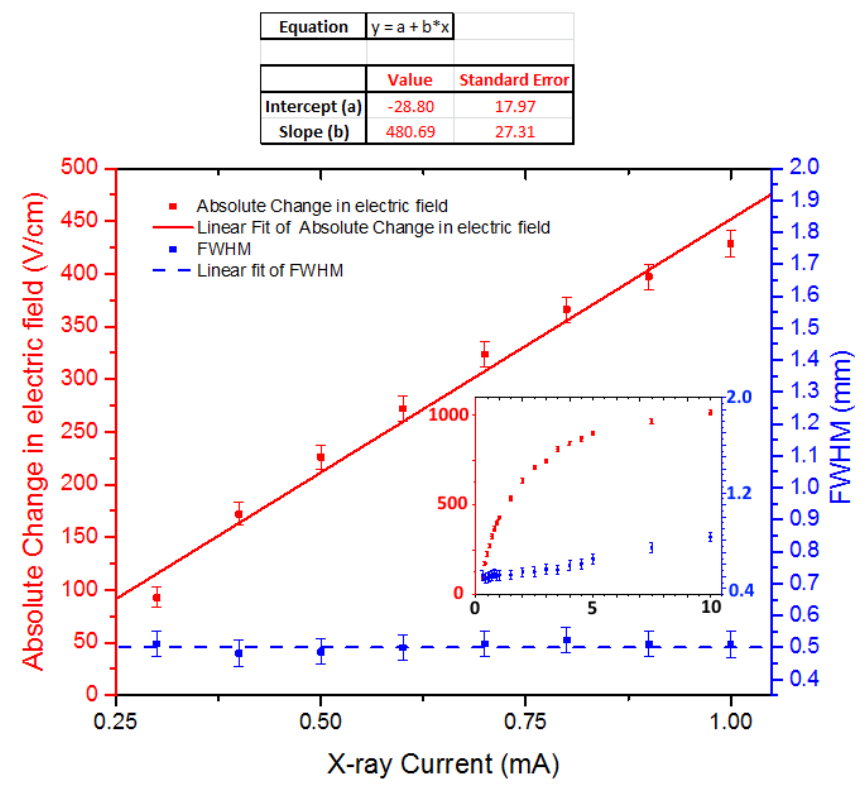

Figure 10: Main: Variation in the depth of intensity dip (measured at $5 \mathrm{~mm}$ from cathode) with increasing X-ray tube current up to $1 \mathrm{~mA}$ (red) under -2.00 $\mathrm{kV}$ bias, the FWHM remains constant (blue). Inset: Same data expanded up to $10 \mathrm{~mA}$.

The plateauing is caused by the field collapsing to within the noise of the system and hence limiting the range, in future experimental setups any signal range limitation may be overcome by running the detector at a different starting bias voltage. 
The X-ray and laser data sets show that there is a clear distinction in location between the signal caused at the charge carrier generation location and space charge build up effects across the whole sample volume, with the latter being focused around the cathode. The effects that are only observed within the charge carrier generation region could be caused by either space charge effects occurring on much shorter timescales, or be directly related to the increase in free charge carriers and hence temporarily reduced resistivity. Our data acquisition time is too long to be sensitive on the timescales comparable to the expected charge carrier transit times that are below milliseconds [4]. Literature suggests that carriers generated locally are likely to not contain a time integrated dose component generated over several milliseconds [15].

The ability to distinguish between the localised effects and those across the whole volume means that the detection beam can be directed so that it passes through the crystal in such a way that only the component generated near the incident radiation is measured. The confirmation of both effects forming part of the detected signal means that any theoretical model method is unlikely to rely solely on the relationship between the number of charge carriers created upon an ionization event and the distortion effect they have on the electric field, but it must also take into account a time integrated element caused by charge trapping. However, at present it is unclear what timescales the two are operating within or which is the dominant mechanism and it is this that will determine the importance it has on any future modeling. In order to disentangle the two effects measurements of the transient after removing NIR/X-ray radiation are planned to be carried out.

\section{CONCLUSIONS}

Our results indicate that the signal measured via the Pockels detection technique is likely to be a combination of trapped space charge beneath the cathode and locally photogenerated carriers. Both effects have been measured under IR laser illumination, where the carriers are released from traps, and from ionizing X-ray irradiation, where the carriers are generated via electron-hole pairs. The results also show that the Pockels approach is sensitive enough to detect small changes in the local electric field caused by varying the beams power and width. However, whilst confirming the presence of both mechanisms by spatially resolving them, the experimental technique used is not capable of discriminating in the time domain and therefore identify conclusively the dominant effect at any given location.

Despite these limitations any theoretical model based on this detection method must combine both components which, owing to the number of variations just within the crystal itself, is complex. Therefore a semi-empirical approach may be most suitable for practical applications. Due to the ability to differentiate between the two effects based on their location, effort should be taken when using this detection technique to focus the detection beam away from the cathode. If this is done then the majority of the detected signal should arise from the local changes which could lead to being able to image the radiation field due to it potentially being an accurate measure of the dose rate from the incident radiation. Polarization within
CZT is common and well documented $[2,4]$ however there are other zinc-blende material options. Both CMT and CdTe may also be suitable for the Pockels technique. CMT has potentially fewer defects due to a lower segregation coefficient and lower levels of manganese in comparison to zinc to achieve the same band-gap energy range (1.5-2.2 eV), however if this comes at the expense of dislocations which are necessary for birefringence then it may not as suitable as CZT [16]. CdTe generally has slightly higher hole carrier mobility than CZT [17] which potentially means space charge becomes less of an issue as fewer holes will be trapped. Investigating these different types of crystals and the effects of Ohmic contacts may provide a signal that has less of a dose component.

As the results indicate the space charge is focused around the cathode, other areas of the crystal may yield a signal with a lower dose component therefore future work will investigate whether the signals response time varies between the areas beneath the cathode and those around the incident beam. A faster readout technique will be used, incorporating a photodiode as the response time is much faster than a CCD camera. This will enable clarification of the time scales that the technique is sensitive to and hence what is being detected.

S. Parsons acknowledges financial support through STFC Grant No. ST/K005669/1.

\section{REFERENCES}

[1] K. A. Nelson, J. A. Geuther, J. L. Neihart, T. A. Riedel, R. A. Rojeski, P. B. Ugorowski, and D. S. McGregor. "Nuclear reactor pulse tracing using a CZT electro-optic radiation detector". Nuclear Instruments and Methods in Physics Research Section A: Accelerators, Spectrometers, Detectors and Associated Equipment, vol. 680, pp. 97-102, Apr. 2012.

[2] G. Prekas, P. J. Sellin, P. Veeramani, A. W. Davies, A. Lohstroh, M. E. Ozsan, and M. C. Veale. "Investigation of the internal electric field distribution under in situ x-ray irradiation and under low temperature conditions by the means of the pockels effect". Journal of Physics D: Applied Physics, vol. 43 (8), Feb. 2010.

[3] K. A. Nelson, N. Edwards, M. J. Harrison, A. Kargar, W. J. McNeil, R. A. Rojeski, and D. S. McGregor. "Investigation of $\mathrm{CZT}$ and $\mathrm{LiNbO} 3$ as electro-optic neutron detectors". Nuclear Instruments and Methods in Physics Research Section A: Accelerators, Spectrometers Detectors and Associated Equipment, vol. 620, pp. 363-367, Feb. 2010.

[4] T. E. Schlesinger, J. E. Toney, H. Yoon, E. Y. Lee, B. A. Brunett, L. Franks, and R. B. James. "Cadmium zinc telluride and its use as a nuclear radiation detector material". Materials Science and Engineering: R: Reports, vol. 32 (4-5), pp. 103-189, Apr. 2001.

[5] A. Cola and I. Farella. "The polarization mechanism in CdTe Schottky detectors". Applied Physics Letters, vol. 94 (10), pp. 102113, March 2009.

[6] A. Lohstroh, D. Blackie, I. Della-Rocca, S. Parsons, A. Langley, and C. Shenton-Taylor, "Cadmium zinc telluride based infra-red interferometry for x-ray detection". Applied Physics Letters, vol. 106, pp. 062597, Feb. 2015.

[7] J. Pousset, I. Farella, S. Gambino, and A. Cola, Journal of Applied Physics, vol. 119, pp. 105701, March 2016.

[8] A. Washington, L. C. Teague, M. C. Duff, A. Burger, M. Groza, and V. Buliga. "Effect of sub-bandgap illumination on the internal electric field of CdZnTe". Journal of Applied Physics, vol. 110 (7), pp. 073708 , Oct. 2011.

[9] A. Cola, I. Farella, N Auricchio and E. Caroli. "Investigation of the electric field distribution in X-ray detectors by Pockels effect". Journal of Optics A: Pure and Applied Optics, vol. 8 (7), pp. S467, Jun. 2006

[10] S. Shwartz, M. Segev, S. Berger, E. Zolotoyabko, and E. ElHanany. "Light-induced ionic polarization in CdZnTe:V 
semiconductor crystals as a source of giant enhancement of nonlinear effects". Physical Review B, vol. 79, pp. 193202, May 2009.

[11] M. Groza, H. Krawczynski, A. Garson, J. W. Martin, K. Lee, Q. Li, M. Beilicke, Y. Cui, V. Buliga, M. Guo, C. Coca, and A. Burger. "Investigation of the internal electric field in cadmium zinc telluride detectors using the Pockels effect and the analysis of charge transients". Journal of Applied Physics, vol. 107 (2), pp. 023704, Jan. 2010.

[12] J. B. Keller. "Diffraction by an Aperture", Journal of Applied Physics, vol. 28 (4), pp. 426-444, Apr. 1957.

[13] A. Cola, I. Farella, A. M. Mancini, W. Dusi, and E. Perillo. "Electric field distribution and charge transport properties in diodelike CdTe X-ray detectors". Nuclear Instruments and Methods in Physics, vol. 568 (1), pp. 406-411, Nov. 2006.

[14] M. Berger, J. Hubbell, S. Seltzer, J. Chang, J. Coursey, R. Sukumar, D. Zucker, and K. Olsen. "NIST Standard Reference Database 8 (XGAM)". Xcom: Photon cross section database (version 3.1), 2010. [Available at: https://www.nist.gov/pml/xcomphoton-cross-sections-database]

[15] M. Prokesch, D. S. Bale, and C. Szeles. "Fast High-Flux Response of CdZnTe X-Ray Detectors by Optical Manipulation of Deep Level Defect Occupations". IEEE Transactions on Nuclear Science, vol. 57 (4), pp. 2397-2399, Aug. 2010.

[16] G. Yang, A. E. Bolotnikov, L. Li, G. S. Camarda, Y. Cui, A. Hossain, K. Kim, V. Carcelen, R. Gul, and R. B. James. "Investigation of Cadmium Manganese Telluride Crystals for Room-Temperature Radiation Detection". Journal of Electronic Materials, vol. 39, pp. 1053-1057, Jul. 2010..

[17] P. J. Sellin, A. W. Davies, A. Lohstroh, M. E. Özsan, and J. Parkin. "Drift Mobility and Mobility-Lifetime Products in CdTe:Cl Grown by the Travelling Heater Method". IEEE Transactions on Nuclear Science, vol. 52 (6), pp. 3074-3078, Dec. 2005. 